\title{
JUURNAL.RU
}

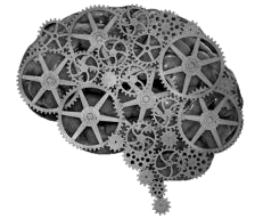

COMPANY GROUP "INTELLEKT"

\author{
Долженко А.М., Рыбалко К.К. \\ Технологический институт (филиал) ДГТУ в г. Азове \\ Азов, Россия
}

doi: 10.18411/lj2016-8-1-06

idsp 000001:lj2016-17-1-06

\section{Уточнение стохастических решений обыкновенных дифференциальных уравнений методом генетическихпреобразований}

Традиционно генетические алгоритмы в качестве входных параметров получают набор случайных величин - хромосому. Это обеспечивает сравнительно медленную сходимость алгоритма[1], а так же возможность попадания в локальный экстремум. Ряд работ авторов[2-5] посвящен анализу возможности применения генетических алгоритмов на конкретном наборе входных параметров - результатах численного решения поставленной задачи классическим алгоритмом. Такой подход позволяет улучшить имеющиеся решения, затратив меньше вычислительных ресурсов по сравнению с чисто генетическим алгоритмом. Данная статья посвящена описанию алгоритма улучшения стохастических решений обыкновенных дифференциальных уравнений методом генетических преобразований.

В качестве входных параметров генетического алгоритмавозьмем вектора $\overrightarrow{\mathrm{Y}}$ и $\overrightarrow{\mathrm{Z}}$ стохастических решений, полученных соответственно методами Эйлера с уточнениями и Рунге-Кутта 4 порядка [6]. Координаты векторов $\overrightarrow{\mathrm{Y}}\left(\mathrm{y}_{1}, \mathrm{y}_{2} \ldots \mathrm{y}_{\mathrm{n}}\right)$ и $\overrightarrow{\mathrm{Z}}\left(\mathrm{z}_{1}, \mathrm{z}_{2} \ldots \mathrm{z}_{\mathrm{n}}\right)$ представляют собой хромосомы, каждый ген которой - это решение в соответствующей точке.

Над хромосомами будем выполнять следующие операции.

1.Скрещивание - получение новой хромосомы, каждый ген которойнаследуетсяот соответствующего гена одного из родителей, выбранных случайным образом из популяции. 
2. Мутация - случайное изменение одного из генов хромосомы. Так как ген - действительное число, то под мутацией гена будем понимать его приращение на величину $\Delta$. В проведенных экспериментах $\Delta$ будет меняться от ничтожно малой до соизмеримой со значением гена.

Входные данные.

Кроме начальных хромосом $\overrightarrow{\mathrm{Y}}$ и $\overrightarrow{\mathrm{Z}}$ (которые входят в популяцию на протяжении всего хода вычислений), на ход выполнения алгоритма влияют следующие параметры.

1.Размер популяции $\mathrm{N}$ - максимальное число хромосом, которое должно быть получено для выбора «наилучшего» решения. Каждая сгенерированная на очередном шаге хромосома может попасть или не попасть в популяцию для дальнейшего скрещивания.

2. Параметр d- задает максимально допустимое отклонение (в \%) от «лучшего» на текущем шаге решения, при котором хромосома будет добавлена в популяцию для дальнейшего скрещивания. Для сравнения решений будем использовать суммы квадратов разностей значений генов хромосомы и соответствующих аналитических значений.

3. Максимально допустимое число итераций - М. Так как при малых значениях dможет потребоваться бесконечно большое число генетических операций для создания гена, удовлетворяющего критерию включения в популяцию, можно задать параметр М, который ограничит вычислительный цикл максимально допустимым числом шагов (a, следовательно, и временем).Мдолжно быть больше или равно N.

4. Диапазон значений, которые может принимать параметр - $\Delta$.

5. Шаг вычислений в стохастических решениях - h.

6 . Вероятность появления мутаций $-\Omega$ (в \%).

Предлагаемый алгоритм состоит из 9 шагов. Приведем его описание.

Шаг 1.

Добавим в популяцию две хромосомы - вектора $\overrightarrow{\mathrm{Y}}$ и $\overrightarrow{\mathrm{Z}}$.

Присвоим текущему размеру популяцииR значение 2.

Выберем в качестве «лучшего» значение хромосомы $\vec{Z}$ (так как метод Рунге-Кутта 4 порядка точнее метода Эйлера с уточнением), присвоим номеру «лучшего» решения $\mathrm{t}$ значение 2. 
Счетчику шагов кприсвоим значение 1

Шаг 2. Пока $\mathrm{R}<\mathrm{Nu} \mathrm{k} \leq$ Мвыполняем шаги 3-8, иначе переходим на шаг 9.

Шаг 3. Генерируем случайным образом два номера хромосом h1и h2из популяции для скрещивания.

Шаг 4. Создаем новую хромосому.

Для каждого гена вновь создаваемой хромосомы генерируем случайное число из набора $\{1,2\}$, которое определяет, ген какого родителя (первого - h1 или второго - h2) попадет на место соответствующего гена в новой хромосоме.

Шаг 5.

Генерируем случайное целое число в диапазоне $\{1 . .100\}$. Если это число меньше либо равно $\Omega$-производим мутацию (шаг 6) иначепереходимнашаг 7.

Шаг 6. Мутация.

Генерируем случайное действительное число $\Delta \mathrm{g}$ в диапазоне $\{1 . . \Delta\}$ и номер мутирующего гена g. Выполняем приращение гена новой хромосомы под номером g на величину $\Delta \mathrm{g}$.

Шаг 7. Увеличиваем счетчик шагов кна единицу.

Находим сумму квадратов разностей значений генов новой хромосомы и соответствующих аналитических значений - S. Если эта сумма не превышает «лучшее» на текущем шаге решение Тболее чем на dпроцентов, то хромосома добавляется в популяцию и выполняется переход на шаг 8, иначе - переход на шаг 2.

Шаг 8. Увеличиваем R на единицу.

ЕслиS $<\mathrm{T}$, то в $\mathrm{T}$ присваиваем $\mathrm{S}$, а в tприсваиваем $\mathrm{R}$.

Переходим на шаг 2.

Шаг 9. Выводим в качестве результирующего значения хромосому под номером $\mathrm{t}$ из созданной популяции.

Описанный выше алгоритм реализован авторами в среде программирования Lazarus. Проведено тестирование алгоритма на различном наборе входных параметров. В качестве примера рассмотрено решение обыкновенного дифференциального уравнения, имеющее простое аналитическое решение: $\mathrm{y}^{\prime}=-2$ упри начальном условии $\mathrm{y}(0)=2$. Очевидно, что решением данного уравнения является функция $\mathrm{y}=2 \mathrm{e}^{-2 \mathrm{x}}$. Проведено сравнение 
решений: аналитического (I), методом Рунге-Кутта 4 порядка (II), методом генетической модификации (III).

Получены следующие результаты:

- разница между решениями Іи ІІІна 99\% меньше разницы между решениями Іи ІІпри значениях $\Omega$ от 3 до 10 , dот 1 до 5 и $\Delta=10-6$, при этом Nвыбиралось в диапазоне от 1000 до 3000, n - в диапазоне от 10 до 30;

- при больших значениях n (100 и выше) разница между решениями Iи III была только на $15 \%$ меньше разницы между решениями Iи II, при этом $\Omega=0,01$, а $\mathrm{d}=3$;

- при значениях n>300 существенного улучшения стохастических решений получено не было.

\section{Выводы.}

Стохастические решения обыкновенных дифференциальных уравнений могут быть существенно улучшены методом генетических преобразований. Описанный выше алгоритм приносит качественно высокий результат при малой степени дискретизации и достаточно небольшом (до 5000) количестве итераций. Точность вычислений составляла 10-10. При увеличении пи Nследует увеличивать точность вычислений.Подобный метод может применяться и для уточнения решений, полученных другими стохастическими алгоритмами. Авторы планируют в ближайшее время проведение исследований на возможность уточнение решений дифференциальных и интегральных уравнений, которые не могут быть получены аналитически. 


\section{Литература:}

1. Долженко А.М., Рыбалко К.К., Робченко М.Н. Сравнение скорости сходимости различных видов генетических алгоритмов / Современные тенденции развития и перспективы внедренияинновационных технологий в машиностроении, образовании и экономике:материалы III Международной научно-практической конференции (Азов, 25 мая2016 г.). / Азов: Изд-во: ООО "АзовПечать", 2016. - 196 стр.

2. Таран В.Н., Долженко А.М., Бойко Е.Ю. Управление и прогнозирование в высшейшколе / Современные проблемы многоуровневого образования: материалы Хмеждународного научно-методического симпозиума (Ростовна-Дону / Дивноморское,2015). - Ростов-н/Д, ДГТУ, 2015.

3. Долженко А.М. Применение генетических алгоритмов при решении задач вэкономике / Информационные технологии в экономических исследованиях: материалынаучно-практической конференции, Ростов-н/Д, ДГТУ, 2013 г.

4. Долженко А.М. Применение генетических алгоритмов на распределенныхвычислительных системах для уточнения стохастических решений классических задачэкономики / Инновационные информационные технологии: материалы международнойнаучнопрактической конференции. Том 4.: МИЭМ НИУ ВШЭ, 2013.

5. Долженко А.М., Бутрина Е.Г. Уточнение решений задачи коммивояжерагенетическими мутациями / Вестник ПГУ. Сер. Математика, механика, информатика. - 2013.- Вып.2(21).

6. Бахвалов Н.С., Жидков Н.П., Кобельков Г.М. Численные методы. 3-е изд., перераб. и доп. М.: БИНОМ. Лаборатория знаний, 2009. 632 с. 